\title{
La Educación en Latinoamérica entre la red y el ecosistema digital, el caso venezolano
}

\section{Latin American Education between the network and the digital ecosystem, the Venezuelan case}

\author{
Ruth Díaz Bello \\ Universidad Central de Venezuela. Venezuela. \\ ruthdiazbello01@gmail.com
}

\begin{abstract}
Resumen
Los cambios que se producen en el ámbito social por el acelerado proceso de innovación tecnológica inciden directamente en el quehacer y la discusión educativa. En el presente trabajo se reflexiona sobre el significado que la interacción en red mediada por las tecnologías de la información y la comunicación (TIC) está teniendo en la vida de los seres humanos. De manera particular se discute sobre la pertinencia de favorecer la integración de los individuos en la red y de cómo las particularidades de la realidad latinoamericana y específicamente la venezolana, pueden afectar el éxito de experiencias educativas y de difusión del conocimiento si no se consideran elementos que conforman el ecosistema digital. A manera de ejemplo, se exponen los factores intervinientes en la toma de decisiones para la realización de EDUWEB 2014, considerando algunos aspectos relevantes del ecosistema digital de la Universidad Central de Venezuela en un momento de crisis con características muy particulares. Se concluye haciendo énfasis en la importancia de la planificación educativa tomando en cuenta políticas internacionales, nacionales e institucionales, destacando de manera especial el valor del compromiso del talento humano con la innovación educativa a partir de los contextos particulares de desempeño del docente y del estudiante a fin de lograr la integración a la red.
\end{abstract}

\section{Palabras clave}

Educación, Interconectividad y red, Políticas Educativas, Ecosistema Digital en Educación

\begin{abstract}
The changes that are produced in the social scope by the accelerated process of technological innovation affect directly in the task and the educational discussion. In this paper, the meaning that the interaction in the networks mediated by the technologies of the information and communication (ICT) are having in the life of the human beings is reflected upon. In particular, the relevance of favoring the integration of individuals in the network is discussed, and how the peculiarities of Latin American reality, and specifically of the Venezuelan one, can affect the success of educational experiences and the knowledge diffusion if some elements that form the digital ecosystem are not considered, such as the variety of services offered and infrastructure in five regions of Venezuela. As an example, the intervening factors in the decision making for the realization of EDUWEB 2014 are exposed, considering some relevant aspects of the Universidad Central de Venezuela digital ecosystem in a moment of crisis with really particular characteristics. The paper concludes by making emphasis in the importance of educational planning considering international, national and institutional politics, highlighting the value of the commitment of the human talent with the educational innovation in a special way, from the particular contexts of the teacher and student performance for the purpose of net integration.
\end{abstract}

\section{Key words}

Education, Interconnectivity and networks Education Policy, Digital Ecosystem in Education. 


\section{La red y la educación}

Hoy día es común reconocer que estamos viviendo cambios acelerados de manera permanente y no se evidencia que a corto plazo esta situación se modifique, por el contrario, se espera que se siga incrementando. Ello lo podemos evidenciar en el rápido desarrollo de la electrónica desde la década de los 70, del siglo XX, cuando surgieron las primeras computadoras personales, las PC, y el posterior proceso de integración tecnológica que ha permitido, que un mismo equipo, le ofrezca a una persona todos los servicios de comunicación e información simultáneamente, en un compacto equipo accesible a la mayoría de la población y con una capacidad de almacenamiento de información prácticamente ilimitada si se hace uso de los recursos disponibles en Internet o como comúnmente se le llama, en la nube.

La oferta de equipos que combinan las opciones de cómputo, acceso a la información y comunicación permanente ha favorecido el crecimiento del mercado de los llamados teléfonos inteligentes, smartphone, y las computadoras portátiles, una variedad de equipos que van integrando servicios y que se confunden hoy día entre teléfonos inteligentes grandes o computadoras portátiles cada vez más pequeñas de pantalla táctil con servicio de telefonía, todos ellos con conexión a Internet.

Esta dinámica del desarrollo tecnológico nos ha colocado sin darnos cuenta, en una sociedad distinta que la Educación, incorporando las tecnologías o no, debe comprender y debe crear espacios de innovación que permitan dar respuestas a las nuevas demandas. Es aún vigente lo planteado por Toffler (1980) cuando señalaba:

Así como la segunda ola [era industrial] combinó el carbón, el acero, la electricidad y el transporte ferroviario para producir automóviles y otros mil productos transformadores de vida, no percibiremos el verdadero impacto de los nuevos cambios hasta que alcancemos el estadio en que se combinen las nuevas tecnologías (...), uniendo computadores, electrónica, materiales nuevos procedentes del espacio exterior y los océanos, con la genética y todo esto, a su vez, con la nueva base energética. La reunión de todos estos elementos liberará un torrente de innovación sin par en la historia humana. Estamos construyendo una tecnosfera dramáticamente nueva para la civilización de la tercera ola (p. 155).

La popularización del término Tecnologías de la Información y la Comunicación (TIC) a partir de la década de los 90 del siglo pasado, fue una muestra de que la clave de este desarrollo estaba en la integración tecnológica, de la cual sólo hemos visto una pequeña parte.

Para efecto del presente trabajo, queremos destacar de manera especial cómo la interconectividad ha favorecido la constitución de un sistema de interacción en red que supera con creces cualquier opción comunicacional que se hubiese poseído hasta el momento y, de manera especial, cómo las limitaciones de los servicios que hay en Latinoamérica y concretamente en Venezuela, incide en el desarrollo de una cultura digital. Se presenta, además, una experiencia desarrollada a nivel universitario para poder llevar a cabo un encuentro académico mediado por TIC.

Las oportunidades que ofrece hoy día la comunicación en red a través de internet haciendo uso de la telefonía móvil está redimensionando el concepto de vida en comunidad, atado inicialmente al plano geográfico, actualmente superado en los espacios virtuales (Castells, 2001; Unesco, 2005; Reig, 2013). 
Las preocupaciones que existen actualmente por incorporar en el proceso educativo la formación en el uso y seguridad en las redes sociales para resguardar la reputación de los estudiantes y de los docentes, favorecer una imagen que esté acorde con el futuro profesional deseado y evitar el bullying o acoso cibernético nos dan indicios del impacto que las tecnologías están teniendo en la constitución del mundo social de los seres humanos y en este caso particular, de los jóvenes.

Igualmente podemos observar la expansión que van teniendo las ofertas para la creación de redes sociales. Más allá de lo que fue inicialmente MySpace (2003) y Facebook (2004), han surgido propuestas para grupos poblacionales específicos como Linkedin (2003), red especializada para la actividad profesional, Twitter (2006) como opción de microbloggings, Instagram (2010) para compartir fotos y videos y, Snapchat (2010) una oferta que está teniendo especial uso por parte de los jóvenes que privilegia el envío de fotos y videos que desaparecen a unos pocos segundos de ser visto. La oferta de aplicaciones para integrarse a las redes sociales de diversos tipos es permanente, incluyendo las que permiten simular vidas virtuales como Second Life (2003).

Las opciones de intercambio en red que se ofrecen en Internet se ven potenciadas por la facilidad de comunicación que brinda la telefonía móvil. El incremento de la conexión a través de equipos móviles está marcando una tendencia importante en el acceso de los jóvenes a las redes.

Reig (2013) hace un detallado análisis sobre el uso del smartphone y lo que denomina el desarrollo del "hiperindividuo" o "individuo conectado", tomando en cuenta para ello el impacto del teléfono en los procesos de socialización e "individualización" de los jóvenes, el desarrollo cognitivo y el desarrollo moral. Ahora bien, el escenario descrito por la autora nos obliga a repensar el término "individuo conectado" para plantear la necesidad de denominarlo "individuo en red". La diversidad de escenarios que se van desarrollando para la dinámica de vida y la oferta de oportunidades para el desarrollo del potencial humano se corresponde no solo con el hecho de estar conectado, sino con el de estar en red.

El término estar conectado pareciera hacer referencia al hecho de tener acceso a información y ofrecer información, pero estar en la red implica participar en dinámicas de interacción multidireccionales, descentralizadas y emergentes, generar información y conocimiento a través de sistemas abiertos de intercambio y colaboración. En estos momentos, más que asegurar una conexión o estar conectado, lo que se busca es integrarse a un entramado de relaciones, de acuerdo a la dinámica de un sistema complejo y abierto en el cual se aporta información y se genera conocimiento. Estar en red demanda: tener conciencia de qué conecta y qué no, durante cuánto tiempo y en qué condiciones. A su vez, demanda reconocer las relaciones de interdependencia que se establecen, qué se acepta y qué no o en qué condiciones y durante cuánto tiempo, a partir del reconocimiento de la diversidad de sujetos y espacios de interacción (intereses, dinámicas, normas, creencias).

Los individuos deben ser capaces de dar respuestas adaptativas en la dinámica de interacción para orientar los cambios y asegurar el equilibrio dinámico del sistema que se ha conformado, sin perder su naturaleza o sentido. En la red se requiere la toma de decisiones permanentes para mantener conexiones, desarrollar nuevas y eliminar las innecesarias a fin de aportar en la dirección deseada.

El comprender que más que individuos conectados la sociedad demanda individuos competentes para desempeñarse de manera idónea en la red, ayuda a redimensionar con 
referentes distintos el proceso educativo. De allí que la tarea supera la acción solitaria del docente que no es nada fácil, porque debe sobreponerse a sus propias limitaciones en el uso de la red para transformarse en un mediador que favorece la formación de un individuo autónomo en estos nuevos espacios, aun en el marco de la autonomía relativa definida por Morin (1991).

De allí la importancia de la práctica innovadora en la labor docente, de la reflexión crítica sobre la práctica y la discusión teórica. Estas son opciones que los educadores tienen a su alcance para, desde la racionalidad y la práctica, orientar una acción educativa que responda al escenario cambiante, de crisis e incertidumbre, en el que se desenvuelve, especialmente atendiendo a las particularidades de los escenarios latinoamericanos. Con la incorporación de las TIC es necesario diferenciar las potencialidades que nos ofrece una herramienta o una propuesta pedagógica de lo que en la práctica educativa se desarrolla debido a las determinaciones que genera el ecosistema digital. Son las acciones, el sentido de las acciones y la significación del contexto en el cual se desarrollan lo que puede favorecer que con una experiencia educativa se logren los objetivos propuestos.

Todo ello requiere la definición de claras políticas públicas dirigidas a favorecer la incorporación de los individuos y ciudadanos al mundo de la red, donde la educación cumple un rol fundamental.

A esta altura es necesario destacar lo más evidente, estar conectados y en red requiere de cuatro elementos fundamentales: infraestructura, equipos personales, conexión a los servicios que proveen internet y un talento humano dispuesto a explorar y usar la oferta de recursos existente. En el escenario que plantea la red, un ecosistema digital de calidad es imprescindible para la integración y la producción de contenidos y servicios. Nos toca entonces preguntarnos ¿Cuáles son las ofertas de servicios en Latinoamérica, especialmente en Venezuela y cómo se pueden usar para responder a los fines educativos?

\section{El ecosistema digital y la educación}

Durante el año 2015 se han presentado informes de gran valor para comprender las características del ecosistema digital de América Latina (CEPAL, 2015.a; CEPAL, 2015.b y Katz, 2015), en ellos se exploran las transformaciones de la industria de las telecomunicaciones y de los servicios basados en Internet para determinar oportunidades y tendencias a nivel comercial y productivo con el desarrollo de la conectividad de alta velocidad y de las tecnologías móviles.

Katz (2015) plantea que el ecosistema digital:

como el conjunto de prestaciones y requerimientos de diversa naturaleza que se proveen desde y a través de las redes de telecomunicaciones, el conjunto de infraestructuras y prestaciones asociadas que habilitan la prestación de dichos servicios, así como la interacción entre los prestadores de servicios de distinta naturaleza que constituyen la cadena de valor extendida de servicios de Internet, constituye un nuevo sujeto de análisis desde el que se debe pensar las políticas públicas (p.XVII).

Para este autor el ecosistema digital involucra tres dimensiones: La primera, tiene que ver con los nuevos modos de producción de información y contenidos y refiere especialmente a "la arquitectura de participación" en la producción; la segunda, a los diferentes comportamientos sociales relativos al uso y consumo de bienes, donde el 
consumidor se transforma en un participante activo en el desarrollo del producto, y la tercera hace referencia a la digitalización, "que describe las transformaciones sociales, económicas y políticas asociadas con la adopción masiva de las tecnologías de información y comunicación.”(p. 16).

Si bien, los indicadores que permiten explorar el ecosistema digital son muy amplios y variados pues atañen a todo el espectro económico, su transformación y su impacto en los ámbitos sociales y de participación política, en el presente trabajo nos vamos a referir a indicadores poblacionales y de servicios que nos remiten a las posibilidades de acceso a Internet y que pueden influir en el desarrollo de actividades educativas mediadas por las TIC, así como al desarrollo de las potencialidades que las mismas ofrecen a los individuos y a los ciudadanos.

Los estudios coinciden en afirmar que en los últimos años la situación de los países de América Latina en relación a la industria de las telecomunicaciones ha mejorado, pero siguen estando muy por debajo de los niveles deseados para lograr la universalización del acceso a Internet:

Entre el 2006 y el 2013, el número de usuarios como proporción de la población en América Latina se ha más que duplicado pasando de 20,7\% a 46,7\%; a pesar de esto, en 2013 el porcentaje de usuarios seguía muy por debajo del de la OCDE (79\%), lo que indica una brecha de 32,3 puntos porcentuales (...) el crecimiento de países como Ecuador y, sobre todo, Venezuela, Colombia y Bolivia fue suficiente para mejorar significativamente su posición en el contexto regional (Katz, 2015; p.30 y 31).

Es importante acotar que, si bien para Venezuela se puede destacar un incremento de usuarios de internet y de velocidad de la banda ancha para el 2015, la heterogeneidad del servicio a lo interno del país es evidente. Para comprender estas diferencias regionales, vamos a ilustrar cómo ello se da considerando, en el caso concreto de Venezuela, las diferencias poblacionales existentes en relación al acceso a internet, disponibilidad de computadora y estabilidad del servicio eléctrico.

Es importante destacar que, para ilustrar las diferencias regionales, se ha utilizado como referencia la ubicación geográfica de los cinco centros regionales que posee la Universidad Central de Venezuela (UCV) para dictar los cursos correspondientes a la carrera de Educación que se imparten a través de los Estudios Universitarios Supervisados (EUS) de la Escuela de Educación. En el Gráfico 1 se observa cómo se distribuyen los centros en la zona norte, nororiental, noroccidental, sur y suroriental del país.

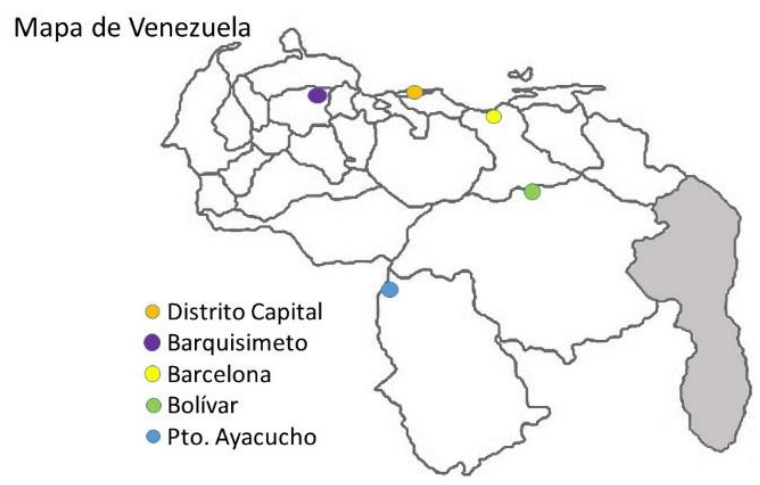


Gráfico 1. Distribución geográfica de los Centros Regionales de los Estudios Universitarios Supervisados de la Escuela de Educación de la UCV.

En la Tabla 1 se puede observar, de acuerdo a los datos arrojados por el XIV Censo Nacional de Población y Vivienda de 2011, que el 23,7\% de los hogares posee acceso a Internet, pero si se revisa la distribución por regiones, se evidencian diferencias importantes. Podemos destacar, primero que los estados Bolívar, Anzoátegui y Lara se encuentran por debajo del promedio nacional entre 1 y dos puntos, pero entre el estado Amazonas y el Distrito Capital, el número de hogares que tienen acceso a Internet en Amazonas, es 4 veces menos que el promedio Nacional y 6 veces menos que el Distrito Capital. En contraste con todas las regiones, el Distrito Capital supera casi el doble del promedio nacional.

\begin{tabular}{|l|c|c|c|c|c|}
\hline \multicolumn{1}{|c|}{ País -Ciudad } & Población & $\begin{array}{c}\text { Acceso a } \\
\text { Internet \% }\end{array}$ & $\begin{array}{c}\text { Computadora } \\
\text { \% }\end{array}$ & $\begin{array}{c}\text { TV por cable } \\
\text { o satélite \% }\end{array}$ & $\begin{array}{c}\text { Telefonía Fija } \\
\%\end{array}$ \\
\hline Venezuela & 27.227 .930 & 23,7 & 31,6 & 48,0 & 50,3 \\
\hline Distrito Capital & 1.943 .901 & 41,5 & 49,8 & 47,1 & 75,1 \\
\hline Bolívar & 1.410 .964 & 21,7 & 29,1 & 48,3 & 43,6 \\
\hline Anzoátegui & 1.469 .747 & 22,7 & 29,2 & 39,5 & 46,7 \\
\hline Lara & 1.774 .867 & 22,8 & 30,5 & 47,1 & 48,8 \\
\hline Amazonas & 146.480 & 5,5 & 15,0 & 35,3 & 27,5 \\
\hline
\end{tabular}

Fuente: Censo Nacional de Población y Vivienda, 2011.

Tabla 1. Disponibilidad de tecnología en el hogar según datos del Censo Nacional de Población y Vivienda, 2011.

En la Tabla 1 se presenta también el porcentaje de hogares con computadoras. A través de este dato se observa la misma tendencia en la distribución que para el acceso a Internet, tres estados que se ubican dentro del rango del promedio nacional (Bolívar, Anzoátegui y Lara), mientras que el Distrito Capital y el estado Amazonas representan los valores extremos, entre el de mayor cantidad de computadoras en el hogar y la menor cantidad, respectivamente. Sin embargo, la distancia entre los valores de las dos entidades disminuye a la mitad en comparación con el acceso.

Así como hay que tomar en cuenta las diferencias poblacionales en relación al acceso a Internet y la disponibilidad de computadoras al momento de desarrollar una propuesta educativa mediada por las TIC, también es necesario considerar las diferencias relacionadas con la velocidad de conexión de los grupos poblacionales a atender. En el caso de Venezuela, de acuerdo al informe de Akamai (2015), se pueden evidenciar estas diferencias de velocidad. En la Tabla 2 se muestra que la velocidad promedio de conexión es de 1.6 Mbps y solamente el 3.6\% de los usuarios tienen conexiones superiores a los 4.0 Mbps. Si bien el incremento de la velocidad se ha producido a nivel nacional, su alcance poblacional es muy bajo. Por otra parte, a nivel porcentual puede 
parecer un incremento muy alto si la velocidad promedio aumentara en el país en un $50 \%$ de la actual, pero la realidad es que, si se produjera un incremento de esa magnitud, no modificaría la posición que tiene Venezuela como el segundo país con la velocidad más baja según el informe de Akamai (2015). De los quince países de América considerados en el informe, Venezuela se encuentra entre los tres países con las velocidades más bajas, solo tres países latinoamericanos tienen velocidades por encima de los 5 Mbps, Uruguay, Chile y México, mientras que Canadá y EEUU tienen velocidades superiores a los $11 \mathrm{Mbps}$.

Las implicaciones que puede tener la velocidad en una conexión de calidad es muy importante debido a que si ésta es deficiente hace que el ambiente de aprendizaje virtual se convierta en un espacio que genera frustración y desagrado en vez de ofrecer facilidades y sensación de logro. Cuando el estudiante debe bajar documentos, subirlos o realizar actividades síncronas como chats con o sin video, debe tener una buena velocidad de conexión que permita que las actividades se lleven a cabo con éxito. Es importante que un docente al planificar actividades educativas mediadas con las TIC valore la capacidad de conexión desde la institución educativa en la que se desempeña, desde su hogar y desde los espacios de conexión de los propios estudiantes para asegurar que la mediación de las TIC realmente favorecerá la experiencia de aprendizaje y no se transformará en un obstáculo en el proceso de enseñanza.

\begin{tabular}{|c|c|c|c|c|c|c|}
\hline Region & $\begin{array}{l}\text { Unique IPVA } \\
\text { Addresses }\end{array}$ & $\begin{array}{c}\text { Average } \\
\text { Connection } \\
\text { Speed (Mbps) }\end{array}$ & $\begin{array}{l}\text { Average Peak } \\
\text { Connection } \\
\text { Speed (Mbps) }\end{array}$ & $\begin{array}{l}\text { \% Above } \\
4 \text { Mbps }\end{array}$ & $\begin{array}{l}\text { \% Above } \\
10 \text { Mbps }\end{array}$ & $\begin{array}{l}\text { \% Above } \\
15 \text { Mbps }\end{array}$ \\
\hline \multicolumn{7}{|l|}{ AMERICAS } \\
\hline Argentina & $7,911,581$ & 4.7 & 25.6 & $42 \%$ & $7.7 \%$ & $1.5 \%$ \\
\hline Bolivia & 294,008 & 1.9 & 16.8 & $3.9 \%$ & $0.3 \%$ & $0.1 \%$ \\
\hline Brazil & $45,956,304$ & 3.6 & 27.0 & $32 \%$ & $2.4 \%$ & $0.6 \%$ \\
\hline Canada & $14,575,081$ & 11.2 & 48.1 & $86 \%$ & $40 \%$ & $17 \%$ \\
\hline Chile & $4,146,700$ & 5.6 & 37.0 & $62 \%$ & $7.4 \%$ & $1.5 \%$ \\
\hline Colombia & $10,810,727$ & 4.5 & 27.8 & $50 \%$ & $2.7 \%$ & $0.6 \%$ \\
\hline Costa Rica & 493,869 & 3.2 & 17.3 & $19 \%$ & $1.1 \%$ & $0.4 \%$ \\
\hline Ecuador & 988,123 & 4.0 & 24.9 & $33 \%$ & $2.5 \%$ & $0.5 \%$ \\
\hline Mexico & $14,171,388$ & 5.5 & 27.3 & $62 \%$ & $6.2 \%$ & $1.7 \%$ \\
\hline Panama & 498,012 & 3.3 & 17.6 & $23 \%$ & $1.2 \%$ & $0.3 \%$ \\
\hline Paraguay & 171,478 & 1.5 & 15.0 & $1.6 \%$ & $0.1 \%$ & $<0.1 \%$ \\
\hline Peru & $1,075,890$ & 4.7 & 28.5 & $55 \%$ & $3.2 \%$ & $0.6 \%$ \\
\hline United States & $148,639,332$ & 11.7 & 50.4 & $77 \%$ & $43 \%$ & $21 \%$ \\
\hline Uruguay & $1,150,495$ & 5.9 & 47.7 & $61 \%$ & $11 \%$ & $28 \%$ \\
\hline Venezuela & $4,299,446$ & 1.6 & 13.9 & $3.2 \%$ & $0.3 \%$ & $0.1 \%$ \\
\hline
\end{tabular}

Fuente: Akamai (2015)

Tabla 2. Promedios de velocidad de conexión en 15 países del continente americano

Para ilustrar las diferencias regionales en Venezuela, podemos tomar como referencia la información de Netindex para el 2012 sobre las velocidades de conexión para las distintas zonas del país. Se puede observar en la Tabla 3, que las diferencias entre las zonas geográficas son significativas y que tanto los valores de subida, como los de 
bajada de información son muy lentos. Como en las tablas anteriores la zona correspondiente al Distrito Capital y en este caso, Barquisimeto, tienen los mejores servicios, con velocidades de conexión superiores al promedio nacional, mientras que el estado Bolívar, de la zona sur del país, tiene los más bajos registros de velocidad, lo que permite suponer que zonas como Puerto Ayacucho, ubicada más al sur, puede tener valores parecidos, tendiendo a ser más bajos. Lamentablemente no se obtuvieron registros para Puerto Ayacucho.

\begin{tabular}{|l|c|c|}
\hline \multicolumn{1}{|c|}{ País -Región } & Download & Upload \\
\hline Venezuela & 1.82 & 0.46 \\
\hline Caracas & 2,88 & 0.73 \\
\hline Ciudad Bolívar & 0.81 & 0.28 \\
\hline Barcelona & 1.25 & 0.39 \\
\hline Barquisimeto & 2.29 & 0.36 \\
\hline Puerto Ayacucho & - & - \\
\hline
\end{tabular}

Fuente: www.netindex.com. Consultado el 1ro de septiembre de 2012

Tabla 3. Promedio de velocidad del servicio doméstico de conexión a Internet

Otro factor, que por elemental no pareciera necesario mencionar, pero que igual afecta la posibilidad de conexión a Internet es el referido a la calidad del servicio eléctrico. En el caso venezolano, la estabilidad y continuidad del servicio eléctrico se ha visto disminuida enlosúltimosaños, lo que hace que ya sea cotidiano que a ciertas horas se vaya la luz. Este problema es menos frecuente en Caracas, la ciudad capital, pero muy frecuente en ciudades como Ciudad Bolívar, Barcelona, Puerto Ayacucho. Al respecto podemos destacar como referencia los resultados obtenidos por Venezuela en el índice de competitividad global elaborado por World Economic Forum (WEF), donde el país ocupa en el año 2015 el puesto 137 entre 144 en el rubro de calidad del suministro eléctrico, con una puntuación de 1.7 en una escala cuyo valor máximo es de 7 puntos, todo ello es indicativo de que en el país existe una alta tendencia a sufrir interrupciones del servicio eléctrico y fallas en las fluctuaciones del voltaje.

Estos datos nos permiten evidenciar la necesidad de que en Latinoamérica se tengan en cuenta las diferencias regionales cuando se programan actividades soportadas en plataformas virtuales o que requieran del uso de las TIC como mediadoras en la relación docente - estudiante. Muchas de estas plataformas se desarrollan teniendo en consideración estándares de servicio que no son necesariamente compatibles con algunos países o localidades de la región. También es importante considerar el incremento de la conexión a internet a través de dispositivos móviles, en el caso venezolano el mayor incremento de conexión a Internet se ha producido por este medio, ello implica que los desarrollos que se realicen a nivel educativo deben dar prioridad a las plataformas con las adecuaciones necesarias para los equipos móviles.

Los docentes que realizan la planificación en los centros educativos que conocen de la disparidad en el acceso a los servicios de su población estudiantil, como en el caso 
particular de los EUS de la UCV, deben responder a las necesidades particulares de las diferentes regiones a las cuales se orientan los programas de formación, de tal manera de no programar actividades que para los estudiantes sean difíciles de cumplir por limitaciones tecnológicas que no están en sus manos resolver. Es por ello que es tan relevante, de acuerdo a lo planteado por Katz (2015), considerar en el ecosistema digital las condiciones de infraestructura y servicio que condicionan la digitalización: los hábitos de uso y las transformaciones que las mismas conllevan en lo económico, político y social.

Ahora bien, en este escenario de cambio permanente e incertidumbre, las universidades tienen el compromiso de asumir los desafíos y seguir avanzando, evaluando las facilidades que puedan ofrecer las políticas públicas, la infraestructura instalada y la calidad de los servicios al alcance de los usuarios para responder a la necesidad de conexión en red. A continuación, presentamos una experiencia que puede ser ejemplo de ello.

\section{EDUWEB 2014, una experiencia acorde con el ecosistema digital}

La Universidad Central de Venezuela, si bien se encuentra en el Distrito Capital, lo que la hace estar en el mejor escenario nacional en lo relativo a conectividad y servicio eléctrico, no está exenta de afrontar dificultades que pueden afectar el desarrollo de actividades académicas mediadas por las tecnologías.

Como ejemplo de ello podemos citar la realización del evento denominado EDUWEB 2014, que la UCV organizó con el apoyo de la Universidad de Carabobo (UC). La universidad organizó y llevó a cabo este evento académico afrontando una realidad institucional con las siguientes características:

1. Cierre por problemas presupuestarios de los programas institucionales de financiamiento para la organización de eventos nacionales e internacionales.

2. Reducción de la velocidad de la banda ancha al $60 \%$ de su capacidad, por problemas de los proveedores del servicio.

3. Dificultad de usar las plataformas disponibles por ser muy demandante de recursos ante la disminución de la velocidad de la banda ancha.

4. Dificultades para obtener divisas que permitieran el pago de honorarios profesionales a los conferencistas invitados.

5. Conflictos a nivel nacional que involucraban al movimiento estudiantil que llevó a evaluar la posibilidad de suspender el evento.

6. Conflictividad gremial a nivel de personal docente, administrativo y obrero que generaba incertidumbre sobre la disponibilidad de los recursos humanos y acceso a los espacios de reunión.

7. Depauperización de los sueldos de los profesores universitarios que limitaba los ingresos por concepto de inscripción en EDUWEB y dificultades para la asistencia al evento.

Considerando esas condiciones, se comenzó a evaluar el escenario existente y considerar estrategias que permitieran afrontar las dificultades detectadas. Para la realización del evento fue necesario: 
1. Contar con el respaldo institucional. La primera de las acciones estuvo dirigida a buscar el respaldo institucional y asegurar que, aunque no se contara con los recursos económicos para financiar el evento, la institución apoyaría a los organizadores con los recursos e infraestructura disponible. En ese sentido se ofrecieron opciones de espacios para la realización de las actividades y se seleccionó el que tenía las mejores condiciones de audio, conectividad y disponibilidad de recurso humano. Estos espacios a su vez fueron acondicionados para asegurar mayor calidad de conexión y se favoreció la velocidad de la misma dándole prioridad a la demanda del evento.

2. Contar con el apoyo del talento humano. Este fue uno de los factores más importantes para la realización del evento, los invitados nacionales e internacionales aceptaron participar ad honorem en la actividad a través de video conferencias, pero posteriormente, ante la baja de la velocidad de conectividad, se le solicitó a los conferencistas que realizaran videos de sus participaciones de tal manera que si la conexión fallaba o el audio no poseía la calidad esperada el día de la presentación, se podía pasar a transmitir el video y luego buscar mecanismos para la interacción con el público y su transmisión vía stream. De igual manera, todo el personal docente y administrativo que le correspondía realizar labores de apoyo tecnológico y de infraestructura, desde las instancias centrales de la Universidad a las de la Facultad y Escuela, atendieron las demandas de los organizadores de manera expedita.

3. Determinar la modalidad a seguir para la realización del evento. En el entendido que los profesores que viven en el interior del país tendrían dificultades para pagar traslados y manutención de tres días en la capital, más el pago de la inscripción, se decidió organizar EDUWEB 2014 bajo una modalidad mixta, con actividades que mezclaban lo presencial con lo virtual. De esta manera se favorecía el hecho de mantener el evento activo, aunque se presentaran actividades gremiales o estudiantiles que pudieran impedir su realización alguno de los días programados. La actividad virtual se alojaría en el servidor destinado a los eventos virtuales de la UCV compartido con actividades de información en los espacios de la UC. Por otra parte, la actividad presencial se realizaría en los espacios físicos de la UCV y se trasmitiría a otras instituciones o personas interesadas a través de la herramienta tecnológica más eficiente de acuerdo a las velocidades de conectividad disponibles para el momento.

4. Seleccionar los recursos de acuerdo a la calidad de los servicios disponibles y de la oferta realizada al público. Mes y medio previo a la realización del evento se probaron diferentes plataformas para determinar la eficiencia del comportamiento de las mismas a la capacidad disponible al momento de hacer las pruebas en el mes de septiembre de 2014. Para este momento la velocidad fue 1.39 Mbps de bajada y $0.46 \mathrm{Mbps}$ de subida dependiendo de la hora (Speedtest.net). Se probaron herramientas como Ustream, Team, Viewer, Hangouts, Blackboard, Skype, Twitcam (Díaz, Almeida, Harvey y Riera, 2015).

El primer día de EDUWEB la velocidad de conexión en el auditórium de la Escuela de Educación de la UCV era de 1.41 Mbps velocidad de bajada y la velocidad de subida 
era $0.47 \mathrm{Mbps}$ (Speedtest.net), con esa velocidad se lograba de manera intermitente mantener la conexión de Skype y trasmitir a través de Twitcam.

El desarrollo exitoso del evento, que implicó ofrecer la oportunidad de escuchar y ver a los ponentes con buena calidad visual y de audio, asegurando la interacción con la misma calidad, requirió del trabajo integrado de un grupo humano que pasaba de un recurso tecnológico a otro de acuerdo al requerimiento comunicacional. El Twitcam funcionó con una laptop, cámara web y micrófono dedicado a la transmisión, mientras la conexión por Skype se mantenía a través de otra laptop y un moderador que informaba a los ponentes sobre lo que sucedía en sala a través del chat de Skype, si no estaban conectados al Twitcam.

Es importante destacar que en los espacios presenciales se contó con el respaldo de todo el personal del Centro Experimental de Recursos Instruccionales (CERI) que daba soporte en las instalaciones de la Sala Anfiteátrica de la Escuela de Educación y se encontraba el personal de apoyo del Campus Virtual de la UCV (SEDUCV) quienes ofrecían orientación a los participantes que tenían poco manejo de las herramientas tecnológicas utilizadas para la presentación de las ponencias, carteles e infografías, ello facilitaba la posterior participación virtual de los asistentes. De igual manera se ofreció apoyo permanente en línea tanto a participantes como ponentes para el uso del Campus Virtual, donde estaban alojadas las ponencias del evento y fueron creados los foros para la discusión de las mismas.

Este apoyo permanente a los asistentes y ponentes facilitaba el acercamiento a la diversidad de herramientas tecnológicas que los participantes no necesariamente habían utilizado anteriormente, pero el entrecruce de los espacios virtuales y presenciales les permitía escoger entre uno y otro de acuerdo a su necesidad y posibilidad de acceso en cada momento del evento.

Los conferencistas se integraron con el equipo organizador de EDUWEB 2014 en la experiencia de favorecer una buena comunicación que permitiera la difusión y discusión de ideas. Algunos expresaban no haber hecho nunca algo parecido, pero participaron y aportaron logrando también un aprendizaje personal.

Cada una de estas experiencias innovadoras generan alternativas educativas y obligan a repensar de manera constante la forma en cómo se deben gestionar los procesos que le son propios a la Universidad en un marco de acción profundamente heterogéneo tanto a nivel de los recursos humanos como de equipamiento tecnológico, accesibilidad y servicio en las diferentes sedes de la universidad, pero que igualmente requiere estar integrados a la red de redes.

\section{Conclusiones}

Toda experiencia de formación o de difusión del conocimiento, que sea mediada por las TIC, debe partir de las posibilidades reales que tiene la población de tener acceso a las mismas, ya que de lo contrario se puede pasar de usar una herramienta con pretensiones de ampliar la cobertura educativa y la difusión del conocimiento pero por las condiciones reales de vida de los estudiantes, transformarse en una vía de exclusión. Ello debe ser especialmente considerado en el caso latinoamericano caracterizado por el desarrollo desigual de sus regiones, tal como ha sido ejemplificado con el caso de Venezuela. 
Tal como lo hemos destacado, no todos los individuos tienen la misma disponibilidad y disposición de acceso a las TIC, nos desenvolvemos en una multiplicidad de escenarios que no son lineales, se yuxtaponen, se entrecruzan y generan nuevas realidades, las culturas se entremezclan y se hacen híbridas en la medida que se producen hibridaciones tecno-sociales. De allí la importancia de valorar el ecosistema digital propio de docentes y estudiantes.

Muchas de las plataformas que se desarrollan con fines educativos están orientadas a facilitar el uso de las tecnologías a profesionales de la docencia que no necesariamente pertenecen a la cultura digital, pero que se acercan a ella para promover experiencias innovadoras de aprendizaje y de difusión del conocimiento mediadas por las TIC.

En términos generales, una plataforma educativa busca de una manera amigable e intuitiva facilitarle al docente la creación de espacios para el intercambio de ideas (por escrito, audio, imágenes fijas o en movimiento), de manera sincrónica o asincrónica, espacios para la publicación de documentos escritos y audiovisuales, y la posibilidad de llevar un control para conocer quiénes, cuánto tiempo y en qué lugares ingresaron a la plataforma además de facilitar espacios para la realización de las evaluaciones.

Todos esos servicios en un solo espacio le permiten al docente resolver todas sus necesidades en un solo entorno, ofreciendo opciones para la discusión y la producción colectiva. Pero una plataforma con una buena oferta de servicios en un ecosistema digital poco eficiente puede transformar la experiencia educativa en algo donde el mensaje es precisamente el contrario al deseado, se coloca tanto a docentes como estudiantes ante una tecnología que no resulta amigable, les hace perder el tiempo, no facilita la comunicación ni la interacción porque la velocidad de la conexión y las interrupciones constantes del servicio generan frustración en cada intento de conexión.

Especialmente en el contexto latinoamericano, el uso de plataformas tecnológicas en una institución educativa, para que sea eficiente, debe partir del conocimiento de la infraestructura y servicios disponibles en la institución, de su eficiencia para el logro de los objetivos educativos que se han propuesto y a partir de allí seleccionar los recursos que mejor respondan a las necesidades a fin de ofrecer una oportunidad educativa motivante, interactiva que permita vivir una experiencia de intercambio y presencialidad, más allá de la distancia.

Es necesario comprender las acciones en sus contextos y en el caso de las acciones educativas en el marco de las políticas que le dan sentido, las instancias organizacionales que se crean para facilitar los procesos formativos, sus propuestas estratégicas, de apoyo y operativas, y evidentemente desde los sentidos personales, profesionales y jerárquicos que interpretan las políticas y orientaciones propuestas. Sin un alto nivel de coordinación las iniciativas que se emprendan estarán destinadas al fracaso.

Por ello Riu (2010) destaca la importancia de la investigación comparada en políticas educativas, debido a que, si bien la globalización ha traspasado las barreras locales, las políticas no pueden tener éxito en contextos diferenciados si se copian de manera acrítica, por la presión por concordar o la imitación ciega:

Los elementos sustantivos de un programa, pese a ser exitosos en un lugar, pueden requerir un mecanismo de aplicación fundamentalmente distinto para ser efectivos en otro. 
... debe subrayarse la importancia de no omitir las complejas y a menudo contradictorias mediaciones nacionales y locales de las tendencias de las políticas "globales", en tanto se forjan otras diferencias en las políticas derivadas de contextos específicos (p. 310).

Puede ser que en un contexto con un ecosistema digital que facilita el uso de las tecnologías solo reste "esperar el paso de una generación para que esas nuevas tecnologías de la información y la comunicación entren de lleno en las costumbres" (Unesco, 2005), pero en un contexto como el latinoamericano, caracterizado por marcadas desigualdades, el paso del tiempo puede generar el incremento de la brecha digital en amplios grupos poblacionales, por ello la actuación inmediata pasa a ser una prioridad.

Los países latinoamericanos presentan entrecruces de pobreza y riqueza, de tecnología de alto nivel en contraste con los vehículos de tracción de sangre, la comunicación icónica-visual en contraposición a la tradición de comunicación oral, la producción industrial en conjunto con la producción de subsistencia. Todo ello obliga a pensar en la necesidad de desarrollar políticas educativas que aseguren la integración de los estudiantes a la cultura digital, unido a la formación de profesionales con altísimo nivel de calidad para el desarrollo de la innovación. Se requiere de respuestas asociadas a las particularidades de los países heterogéneos e híbridos que confrontan la mezcla de todos los niveles del desarrollo de la humanidad en un solo territorio y en espacios contiguos (García, 1989).

Desde la experiencia de la UCV, se puede decir que se ha asumido desde sus diferentes niveles organizacionales el reto de innovar haciendo uso de las TIC, sin embargo cada uno de los niveles institucionales ha tenido que enfrentar diversidad de obstáculos asociados a la prestación de servicio, el equipamiento tecnológico, las restricciones presupuestarias, la capacidad de respuesta institucional y las particularidades de los actores involucrados (Harvey, 2010 y Díaz, 2012). Esto nos lleva a la importancia de diseñar políticas claras y flexibles para las instituciones educativas, reconociendo que las políticas no van a demarcar una instrucción con líneas de acción que todos los integrantes de la organización van a seguir sin discusión ni interpretaciones personales o posiciones diferenciadas. Una institución universitaria recoge multiplicidad de visiones que a su vez responden a intereses diferentes pero la construcción conjunta de políticas institucionales favorece un compromiso con la institución y sus políticas.

Se requiere que los términos y acuerdos que dan soporte a las políticas se sustenten en una seria discusión teórica para evitar que la construcción de propuestas, las acciones promovidas y ejecutadas se realicen sobre discursos vacíos, elaborados a partir de frases hechas y "homologadas" de las cuáles no se tiene muy claro su contenido y consecuencias institucionales y que en ocasiones descuidan lo pedagógico. El diseño de políticas educativas no es sólo un problema de cubrir trámites burocráticos, está orientado a la definición de hacia dónde quiere ir una institución y una sociedad y el rol que la educación cumple en ello.

Las propuestas que se concretan en la planificación se enmarcan en un modelo de sociedad que se desea alcanzar y que se estima posible, una respuesta de una organización que se construye alrededor de un objetivo compartido por la comunidad que la conforma. De allí que los diferentes niveles de la planificación, nacional, 
institucional o de aula, nos muestran una posibilidad de hacer realidad los objetivos propuestos en un tiempo determinado, a pesar de las adversidades. Para su realización es condición indispensable considerar los diferentes aspectos que conforman el ecosistema digital, pero de manera especial, el talento humano formado $\mathrm{y}$ comprometido con la institución y su visión de futuro. En el caso particular referido en el presente trabajo, se trata de buscar vías para generar los compromisos y acciones dirigidos a la discusión teórica y la innovación tecnológica para lograr estar en red, dando énfasis en el rol de los docentes y los alumnos en los escenarios en los cuales se encuentran

Presentación del artículo: 8 de noviembre de 2015

Fecha de aprobación: 9 de diciembre de 2015

Fecha de publicación: 30 de enero de 2016

Díaz-Bello, R. (2016). La La Educación en Latinoamérica entre la red y el ecosistema digital, el caso venezolano. RED. Revista de Educación a Distancia. 48(8). Consultado el (dd/mm/aaaa) en http://www.um.es/ead/red/48/ruth.pdf

\section{Bibliografía}

Akamai (2015). Akamai's state of the internet. Q2 2015 report . Recuperado el 21-112015 de https://www.akamai.com/us/en/multimedia/documents/content/akamai-stateof-the-internet-report-q2-2015.pdf

Castells, Manuel (2001). La galaxia Internet. Reflexiones sobre Internet, empresas y sociedad. Barcelona: Plaza \& Janés Editores, S.A.

CEPAL (2015.a). La nueva revolución digital. De la Internet del consumo a la Internet de la producción. Chile: Naciones Unidas. Julio, 2015. Recuperado de http://repositorio.cepal.org/bitstream/handle/11362/38604/S1500587_es.pdf?sequenc $\mathrm{e}=1$.

CEPAL (2015.b). Estado de la banda ancha en América Latina y el Caribe. Naciones Unidas, Santiago de Chile, julio de 2015.

Díaz Bello, R. (2012). Oportunidades de la realidad virtual en diferentes escenarios geográficos. Simposio La realidad virtual y la diversidad de escenarios educativos En: EDUTEC 2012. Canarias en tres continentes digitales: educación, TIC, NETCoaching. Las Palmas de Gran Canaria (España). Universidad de Las Palmas de Gran Canaria. 14 al 16 de noviembre de 2012. Memorias Digitales del Evento. Recuperado de http://www.edutec.es/sites/default/files/congresos/congresoedutec12.pdf pag. 109

Díaz, R. et al. (2015). Experiencias Híbridas con TIC: una alternativa en realidades complejas. En: EDUTEC. XVIII Congreso Internacional. Riobamba, Ecuador. 
EDUWEB 2014. Décima Expedición EDUWEB 2014. Retos Educativos: El conocimiento, los jóvenes y la emprendeduría. Recuperado de http://www.eduweb.org.ve/eduweb2014/

García Canclini, N. (1989). Culturas Híbridas, estrategias para entrar y salir de la modernidad. México: Grijalbo.

Harvey, I. (2010). Desarrollo de una propuesta de gestión de la innovación en la práctica educativa apoyada en las TIC para la Escuela de Educación de la UCV. Tesis de Maestría publicada, Universidad Central de Venezuela, Caracas.

Katz, R. (autor -coordinador) (2015). El ecosistema y la economía digital en América Latina. Barcelona: Ariel, y Fundación Telefónica. Recuperado de http://www.fundaciontelefonica.com/arte_cultura/publicaciones-listado/pagina-itempublicaciones/?itempubli=430.

Morin, E. (1991). Cultura n conocimiento. En: p. Watzlawick y P. Krieg (Comp.). El ojo del observador. Barcelona: Gedisa.

Netindex (2012) Consultado el 1ro de septiembre de 2012. Recuperado de http://www.netindex.com.

Reig, D. (2013). Describiendo al hiperindividuo, el nuevo individuo conectado. En: Reig, Dolors y Vílchez, Luis F. (2013). Los jóvenes en la era de la hiperconectividad: tendencias, claves y miradas. Madrid: Fundación Telefónica y Fundación Encuentro.

Rui, Y. (2010). "La comparación de políticas” En: Bray, M.; Adamson, B. y Mason, M (compiladores) (2010). Educación comparada. Enfoques y métodos. Buenos Aires: Granica.

República Bolivariana de Venezuela. Ministerio del Poder Popular del Despacho de la Presidencia. Instituto Nacional de Estadística. XIV CENSO NACIONAL DE POBLACIÓN Y VIVIENDA. Resultados por Entidad Federal y Municipio del Distrito Capital. Agosto, 2012.

República Bolivariana de Venezuela. Ministerio del Poder Popular del Despacho de la Presidencia. Instituto Nacional de Estadística. XIV CENSO NACIONAL DE POBLACIÓN Y VIVIENDA. Resultados por Entidad Federal y Municipio del Estado Lara. Agosto, 2012. Recuperado el 2/09/2012 de http://www.ine.gov.ve/documentos/Demografia/CensodePoblacionyVivienda/pdf/lar a.pdf

República Bolivariana de Venezuela. Ministerio del Poder Popular del Despacho de la Presidencia. Instituto Nacional de Estadística. XIV CENSO NACIONAL DE POBLACIÓN Y VIVIENDA. Resultados por Entidad Federal y Municipio del Estado Anzoátegui. Agosto, 2012. Recuperado el 2/09/2012 de http://www.ine.gov.ve/documentos/Demografia/CensodePoblacionyVivienda/pdf/an zoategui.pdf 
República Bolivariana de Venezuela. Ministerio del Poder Popular del Despacho de la Presidencia. Instituto Nacional de Estadística. XIV CENSO NACIONAL DE POBLACIÓN Y VIVIENDA. Resultados por Entidad Federal y Municipio del Estado Bolívar. Agosto, 2012. Recuperado el 2/09/2012 de http://www.ine.gov.ve/documentos/Demografia/CensodePoblacionyVivienda/pdf/bol ivar.pdf

República Bolivariana de Venezuela. Ministerio del Poder Popular del Despacho de la Presidencia. Instituto Nacional de Estadística. XIV CENSO NACIONAL DE POBLACIÓN Y VIVIENDA. Resultados por Entidad Federal y Municipio del Estado Amazonas. Agosto, 2012. Recuperado el 2/09/2012 de http://www.ine.gov.ve/documentos/Demografia/CensodePoblacionyVivienda/pdf/A mazonas.pdf

República Bolivariana de Venezuela. Ministerio del Poder Popular del Despacho de la Presidencia. Instituto Nacional de Estadística. Resultados Básicos. Censos Básicos. Caracas, 9-08-2012. Presentación Power Point. Recuperado el 2/09/2012 de http://www.ine.gov.ve/documentos/Demografia/CensodePoblacionyVivienda/pdf/Re sultadosBasicosCenso2011.pdf

Toffler, A. (1980). La tercera ola. España: Plaza \& Janes.

UNESCO (2005). Hacia las sociedades del conocimiento. Informe mundial de la Unesco. Paris: Unesco. Recuperado de http://unesdoc.unesco.org/images/0014/001419/141908s.pdf.

World Economic Forum (WEF) (2015) The Global Gender Gap Report 2015. 10th Anniversary Edition. Recuperado el 19-11-2015 de http://reports.weforum.org/global-competitiveness-report-20142015/economies/\#indexId=GCI\&economy=VEN 